Ann. Zootech., I973, 22 (I), 83-92.

\title{
EFFET DE LA TENEUR EN PROTÉINES DE RATIONS A BASE DE SOJA OU DE SESAME SUR LA CROISSANCE DU LAPIN
}

\author{
F. LEBAS \\ avec la collaboration technique de Marie-Claude Cousin et G. SARdi \\ Station de Recherches sur l'Élevage des Porcs, \\ Centre national de Recherches zootechniques, I. N.R. A., \\ 78350 Jouy en Josas \\ RÉSUMÉ
}

Au cours de 3 expériences successives, nous avons évalué sur un total de I7o lapins en croissance, l'efficacité de régimes à taux protéiques variables. Dans toutes les rations les protéines ont été apportées uniquement par du tourteau de soja (I I à 29 p. Ioo de protéines) ou du tourteau de sésame (1 3 à 25 p. roo de protéines). Pour les 2 types de protéines la croissance est améliorée jusqu'à un seuil de I 7-I 8 p. ıoo de protéines. L'indice de consommation est amélioré avec le soja jusqu'aux environs de i 8-20 p. roo de protéines. En revanche, avec le sésame, l'indice de consommation a été pratiquement constant pour les taux de protéines variant de I 3 à 25 p. Ioo. L'étude de la composition en acides aminés de nos rations nous laisse penser que les besoins en lysine donnés par la littérature sont probablement très largement surestimés.

\section{INTRODUCTION}

Les études menées jusqu'à présent sur le besoin azoté du lapin en croissance ont essentiellement porté sur les aspects quantitatifs globaux (BRADFIELD et MAYNARD, I958; Hofmann et Steiner, I959; Smith, DonzFer et Mathied, I960; HeCKMANN et MeHner, I970). Ces différents auteurs ont utilisé des rations complexes dans lesquelles les proportions des différentes sources azotées changent pour chaque taux de protéines utilisé. Cette pratique se traduit par une modification de la composition de la protéine moyenne. Or, depuis les travaux de MC WARD, Nicholson et Poulton (I967), Gaman et Fisher (I970), Cheeke (I971), Adamson et Fisher 
(I97I), on sait que le lapin en croissance est sensible à la qualité des protéines. Dans ces conditions, il nous a semblé utile d'aborder le problème du taux optimum de protéines en fonction de la nature de la protéine considérée, celle-ci étant la même quel que soit le niveau azoté de la ration. Nous rapportons ci-dessous le résultat de différentes expériences où nous avons fait varier le taux de protéines de rations comportant du tourteau de soja ou de sésame comme seule source azotée.

\section{MATÉRIEL E'T MÉTHODES}

Trois expériences successives ont été réalisées. Les deux premières (A et B) correspondent chacune à 5 taux d'incorporation de tourteau de soja et la troisième (C) à 5 taux de tourteau de sésame.

\section{A. - Les animaux}

Les trois expériences ont été réalisées au moyen de I 70 lapereaux Californien des 2 sexes, àgés de 5 semaines au départ. Les expériences ont duré 5 semaines. Tous les animaux ont été élevés dans des cages individuelles entièrement métalliques à l'intérieur d'un bâtiment décrit précédlemment (LEBAS, I968).

\section{B. - Les contrôles}

La consommation alimentaire a été estimée par différence entre les quantités distribuées et les refus, chaque jour de semaine. Les animaux ont été pesés I fois chaque semaine, à la même heure, durant toute l'expérience. Les teneurs en matière sèche, minéraux et azote de tous les aliments ont été déterminées, de même que la composition en acides aminés des tourteaux utilisés.

\section{C. - Dosages chimiques}

Les teneurs en matière sèche ont été obtenues après dessiccation 24 heures à I $^{\circ} 3^{\circ} \mathrm{C}$. Les cendres ont été obtenues sur les mêmes échantillons après incinération pendant $\mathrm{I}_{4}$ heures, à $55^{\circ} \mathrm{C}$. Les protéines ont été estimées en multipliant par 6,25 la quantité d'azote dosée par la méthode Kjeldhal. Les acides aminés ont été closés par chromatographic sur colonne, grâce à un appareil automatique JEOL JLC - 5 AH.

\section{D. - Les régimes des 3 expériences}

L'expérience A a porté sur 60 animaux. Ceux-ci ont reçu comme aliment unique l'un des 5 régimes comprenant théoriquement I I, I4, I7, 20 ou 23 p. Ioo de protéines provenant du soja (tabl. I). L'expérience B correspond à la suite de l'expérience A. Aussi, les 70 lapins correspondant, ont-ils reçu des régimes comprenant théoriquement $17,20,23,26$ ou 29 p. Ioo de protéines provenant du soja (tabl. I). Pour l'expérience $\mathrm{C}$ enfin, nous avons distribué aux 40 lapins l'un des 5 régimes à base de tourteau de sésame comportant théoriquement I3, I6, I9. 22 ou 25 p. Ioo de protéines (tabl. I). Les tableaux 2 et 3 donnent la composition chimique sommaire des différents régimes utilisés. A partir de la composition en acides aminés des deux tourteaux utilisés et de la formulation des régimes, nous avons calculé la composition en acides. aminés des différents aliments (sans tenir compte de l'apport de la paille). Les résultats de cette approximation sont consignés au tableau 4 pour la lysine, l'arginine et les acides aminés soufrés $\left({ }^{1}\right)$.

(1) Seuls ont été pris en considération ces 4 acides aminés car à la date de réalisation de l'expérience, on ignorait encore la liste des acides aminés indispensables aux lapins, publice depuis par ADAMSON et Fisher (r97i). 
TABLEAU I. - Composition des différents régimes utilisés au cours des expériences $A, B$ et $C$

\begin{tabular}{|c|c|c|c|c|c|c|c|c|c|c|c|c|}
\hline \multirow{2}{*}{ Régimes } & \multicolumn{7}{|c|}{ Soja } & \multicolumn{5}{|c|}{ Sésame } \\
\hline & 11 & $1 / 4$ & 17 & 20 & 23 & 26 & 29 & 13 & 16 & 19 & 22 & 25 \\
\hline $\mathrm{T}_{x}$ sоја $\ldots$ & 20 & 26 & 32 & 38 & 44 & 50 & 56 & - & - & - & - & - \\
\hline $\mathrm{T}_{\boldsymbol{x}}$ sésame .... & 一 & - & - & - & - & - & - & 28,5 & 35,5 & 42,5 & 49,5 & 56,5 \\
\hline Amidon maïs. & 53 & 47 & 11 & 35 & 29 & 23 & 17 & 4,5 & 37,5 & 30,5 & 23,5 & 16,5 \\
\hline Huile maïs ... & 3 & 3 & 3 & 3 & 3 & 3 & 3 & 3 & 3 & 3 & 3 & 3 \\
\hline Paille orge ... & 20 & 20 & 20 & 20 & 20 & 20 & 20 & 20 & 20 & 20 & 20 & 20 \\
\hline C. M. V. . . . & 4 & 4 & 4 & 4 & 4 & 4 & 4 & 4 & 4 & 4 & 4 & $\dot{\prime}^{\prime}$ \\
\hline
\end{tabular}

TABLEAU 2. - Composition chimique des régimes utilisés dans les expériences $A$ et $B$

\begin{tabular}{l}
\hline \\
Régimes
\end{tabular}

TABLEAU 3. - Composition chimique des rógimes utilisés pour l'expérience C

\begin{tabular}{|c|c|c|c|c|c|}
\hline \multirow{2}{*}{ Régimes } & \multicolumn{5}{|c|}{ Sésame } \\
\hline & 13 & $16 j$ & 19 & 22 & 25 \\
\hline Matière sèche (p. 100) . & 88,0 & 89,0 & 88,6 & 89,5 & 89,5 \\
\hline Protéines $(\mathrm{N} \times 6,25)($ p. 100$) \ldots$ & 13,0 & 16,0 & 18,5 & 22,6 & 24,8 \\
\hline Cendres (p. 100) ........... & 8,3 & 10,1 & 10,6 & 12,5 & 13,2 \\
\hline
\end{tabular}

TABIEAU 4. - Composition en différents acides aminés calculée pour les régimes utilisés dans les expériences $A, B$ et $C$

\begin{tabular}{|c|c|c|c|c|c|c|c|c|c|c|c|c|}
\hline \multirow{2}{*}{$\begin{array}{c}\text { Acides aminés } \\
\text { en p. } 100 \\
\text { du régime }\end{array}$} & \multicolumn{7}{|c|}{ Soja } & \multicolumn{5}{|c|}{ Sésame } \\
\hline & 11 & $1^{\prime} t$ & 17 & 20 & 23 & 26 & 29 & 13 & 16 & 19 & 22 & 25 \\
\hline Arginine . & 0,65 & 0,85 & 1,04 & 1,24 & 1,43 & 1,63 & 1,82 & 1,16 & 1,94 & 2,31 & 2,67 & 3,03 \\
\hline Lysine . . . . . & 0,63 & $0,8 \geq$ & 1,01 & 1,20 & 1,39 & 1,58 & 1,77 & 0,37 & 0,45 & 0,54 & 0,62 & 0,71 \\
\hline Méthionine ... & $0,1 / 4$ & 0,18 & 0,22 & 0,27 & 0,31 & 0,35 & 0,39 & 0,35 & 0,44 & 0,52 & 0,60 & 0,68 \\
\hline Cystine ..... & 0,15 & 0,20 & 0,24 & 0,29 & 0,33 & 0,38 & 0,12 & 0,27 & 0,35 & 0,41 & 0,48 & 0,55 \\
\hline
\end{tabular}




\section{F. -- Exploitation des résultats}

Les 3 expériences ont été conduites selon un schéma en blocs complets équilibrés. Les comparaisons ont été faites, au scin de chaque séric expérimentale, grâce à une analyse de variance et à un test de Tukey, pour comparer les moyennes après l'analyse précédente.

\section{RÉSULTATS}

\section{A. -- Expérience $A$}

Les résultats généraux de la première expérience montrent qu'il n'y a plus d'augmentation significative des performances de croissance ou de consommation au-dessus de I7 p. Ioo de protéines (tab1. 5) ; par contre, l'indice de consommation semble se stabiliser mais avec moins de netteté, aux environs de I7-20 p. roo de protéines. Il convient de remarquer le niveau satisfaisant des performances tant de croissance que pour l'utilisation des aliments ; en effet, même pour le régime le plus pauvre en protéines et bien que la croissance ait été relativement faible $(20 \mathrm{~g} / \mathrm{j})$ par suite d'une consommation d'aliment réduite, l'indice de consommation est modéré.

\section{TABLEAU 5}

Performances moyennes des lapins au cours de l'expérience $A$

\begin{tabular}{|c|c|c|c|c|c|}
\hline Régime soja & 11 & 14 & 17 & 20 & $\geq 3$ \\
\hline $\begin{array}{l}\text { Consommation } \\
\text { d'aliment }(g / \mathrm{i}) \\
\qquad \bar{x} \pm s_{\bar{x}}\end{array}$ & $70,3 \pm 3,0^{a}$ & $80,70 \pm 3,4 b$ & $102,9 \pm 2,2 c$ & $106,1 \pm 4,7^{\circ}$ & $109,7 \pm 5,4 c$ \\
\hline $\begin{array}{c}\text { Gain de poids } \\
\text { moyen }(g / \mathbf{j}) \\
\bar{x} \pm s^{-}\end{array}$ & $20,1 \doteq 1,5 a$ & $26,6 \pm 2,0^{b}$ & $33_{1}, 2 \pm 0,7 c$ & $36,8+1,8^{c}$ & $38,41 \pm 2,3^{c}$ \\
\hline $\begin{array}{c}\text { Indice de consom. } \\
\bar{x}+s_{\bar{x}}\end{array}$ & $3,61 \pm 0,18^{\alpha}$ & $3,40 \pm 0,32 a b$ & $3,0 \mathrm{0} \pm 0,0+b c$ & $2,91 \pm 0,06^{c}$ & $2,91 \pm 0,11^{c}$ \\
\hline
\end{tabular}

$a, b, c:$ Les movennes portant une lettre différente en indice différent significativement au seuil $\mathrm{P}<0,05$.

Le tracé de la courbe de croissance des animaux pour les différents régimes (fig. I) permet de préciser l'évolution régulière du poids vif des lapins des 5 lots expérimentaux durant les 5 semaines d'expérience. On peut parallèlement remarquer que les courbes correspondant aux régimes à II et I4 p. Ioo de protéines se distinguent nettement du faisceau de courbes correspondant aux 3 autres types d'alimentation dès les premières semaines, en particulier pour le régime le plus pauvre. 


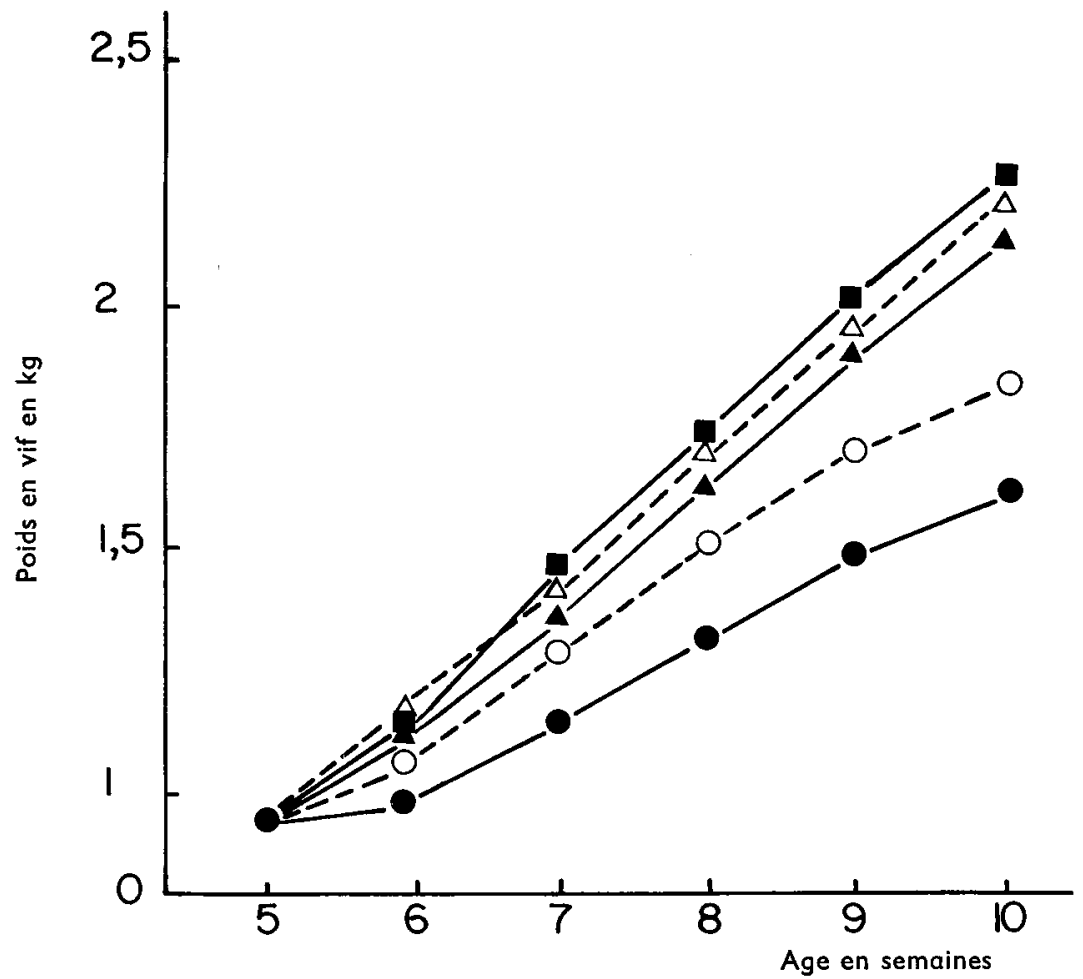

FIG. I. - Évolution du poids vif des lapins au cours de l'expérience $A$, pour les différents régimes à base de soja

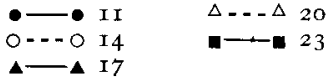

TABLEAU 6

Performances moyennes des lapins au cours de l'expérience $B$ (aucune des différences n'est significative)

\begin{tabular}{c|c|c|c|c|c}
\hline Régime soja & 17 & 20 & 23 & 26 & 29 \\
\hline $\begin{array}{c}\text { Consommation } \\
\text { d'aliment }(\mathrm{g} / \mathrm{j})\end{array}$ & $95,3 \pm 2,5^{*}$ & $95,1 \pm 3,0$ & $97,4 \pm 3,5$ & $98,7 \pm 4,2$ & $101,8 \pm 4,7$ \\
\hline $\begin{array}{c}\text { Gain de poids } \\
\text { moyen }(\mathrm{g} / \mathrm{j})\end{array}$ & $32,3 \pm 1,4$ & $33,6 \pm 1,4$ & $33,0 \pm 1,6$ & $32,4 \pm 1,2$ & $34,3 \pm 2,0$ \\
\hline \begin{tabular}{c} 
Indice de consom. \\
\hline
\end{tabular} & $2,99 \pm 0,09$ & $2,84 \pm 0,0 !$ & $2,98 \pm 0,07$ & $3,05 \pm 0,07$ & $3,16 \pm 0,10$ \\
\hline
\end{tabular}

$* \bar{x} \pm s \bar{x}$. 


\section{B. - Expérience $B$}

Au cours de cette expérience avec des régimes contenant de 17,7 à $28,7 \mathrm{p}$. IoO de protéines, nous n'avons enregistré aucune différence dans les performances des animaux affectés aux divers lots (tabl. 6). Les consommations et les vitesses de croissance pour les lots correspondant sont légèrement inférieures à celles enregistrées lors de l'expérience $A$. Les indices de consommation, par contre, sont du même ordre de grandeur. A ce propos, il convient de souligner la légère augmentation de l'indice de consommation pour les teneurs en protéines du régime les plus élevées bien que les différences ne soient pas significatives.

\section{C. - Expérience $C$}

Nous retrouvons avec le sésame des résultats (tabl. 7) comparables à ceux enregistrés précédemment avec le soja lors de l'expérience $\mathrm{A}$. A pa-tir du régime sésame ig (I8,5 p. Ioo de protéines) les performances de croissance et de consommation ne sont plus augmentées. Par contre l'indice de consommation, même pour les régimes les plus pauvres, a été pratiquement constant pour tous les taux de protéines utilisés $(3,40 \pm 0,09)$. On peut donc constater que les différences entre les vitesses de croissance sont dues aux variations dans l'ingestion d'aliment. L'expérience avec le sésame se distingue donc de la première expérience avec le soja; en effet, dans une gamme proche de taux de protéines, l'indice de consommation était amélioré, jusqu'à un certain seuil, par une élévation de la teneur en protéines de la ration, ce phénomène ne se retrouve plus avec le sésame.

TABIEAU 7

Performances moyennes des lapins au cours de l'expérience $C$

\begin{tabular}{c|c|c|c|c|c}
\hline \hline Régime sésame & 13 & 16 & 19 & 21 & 25 \\
\hline $\begin{array}{c}\text { Consommation } \\
\text { d'aliment }(\mathrm{g} / \mathrm{j})\end{array}$ & $85,0 \pm 2,5^{a *}$ & $88,8 \pm 3,9^{a}$ & $107,2 \pm 4,1^{b}$ & $112,0 \pm 3,4^{b}$ & $117,7 \pm 5,9^{b}$ \\
\hline $\begin{array}{c}\text { Gain de poids } \\
\text { moyen }(\mathrm{g} / \mathrm{j})\end{array}$ & $24,0 \pm 1,3^{a}$ & $26,0 \pm 1,1^{a}$ & $33,1 \pm 1,2^{b}$ & $34,1 \pm 1,6^{b}$ & $34,1 \pm 2,1^{b}$ \\
\hline \begin{tabular}{c} 
Indice de consom. \\
\hline
\end{tabular} & $3,59 \pm 0, \mathbf{1}^{a}$ & $3,4^{2} \pm 0,07^{a}$ & $3,21 \pm 0,05^{a}$ & $3,31 \pm 0,06^{a}$ & $3,4^{\prime} 8 \pm 0,10^{a}$ \\
\hline
\end{tabular}

$a, b$ : Les moyennes portant une lettre différente en indice diffèrent significativement au seuil $\mathbf{P}=0,05$.

$*: \bar{x} \pm s_{\bar{x}}$.

\section{D. - Effet du taux de protéines}

Afin de faciliter la discussion des résultats expérimentaux nous avons réuni aux figures 2 et 3 , pour les trois expériences, les vitesses de croissance et les efficacités protidiques en fonction du taux de protéines de la ration. Ainsi, la représen- 


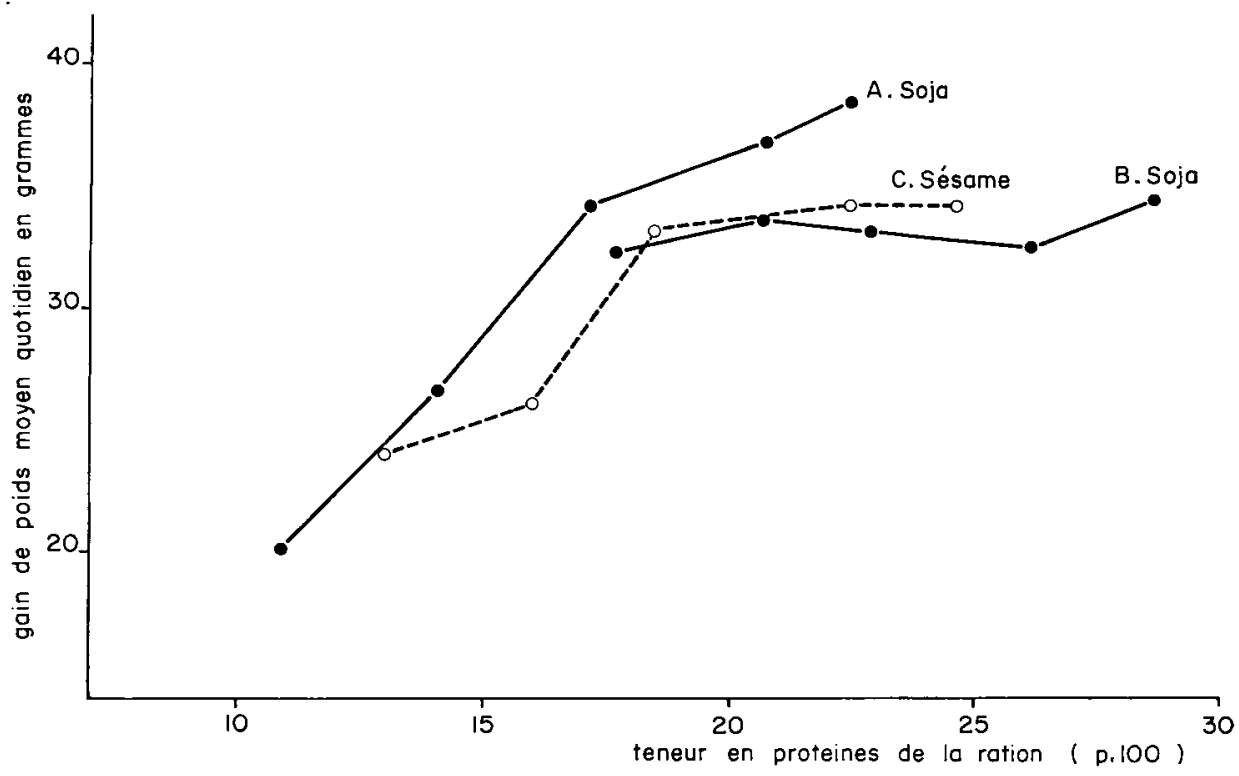

FIG. 2. - Variation de la vitesse de croissance des lapins en fonction de la teneur en protéines de la ration au cours des expériences $A$ et $B$ (soja) ainsi que $C$ (sésame)

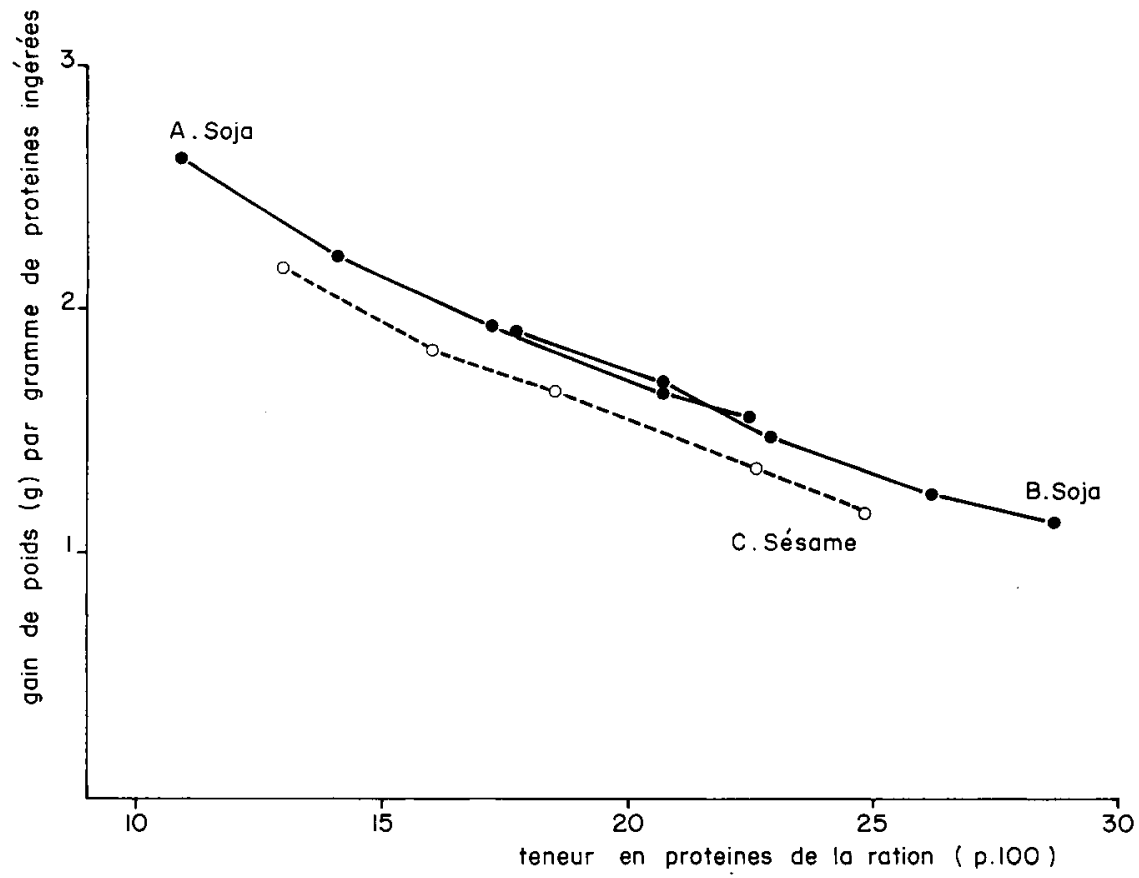

FIG. 3. - Évolution de l'efficacité protidique en fonction de la teneur en protéines de la ration au coutrs des expériences $A$ et $B$ (soja) ainsi que $C$ (sésame) 
tation graphique de la figure 2 permet de souligner l'augmentation de la vitesse de croissance tant que la teneur en protéines n'a pas atteint un seuil d'environ I7-I8 p. Ioo de protéines; ceci est valable pour les 2 protéines utilisées. Au-delà de ce seuil, et jusqu'aux environs de 29 p. Ioo de protéines, la vitesse de croissance reste constante.

Par contre, l'augmentation du taux de protéines entre I I et 29 p. Ioo entraîne une diminution régulière de l'efficacité protidique (fig. 3). Cette diminution est probablement du type hyperbolique et semble de même nature pour le soja et le sésame. Cependant, pour des taux équivalents, il existe une différence d'environ $0,2 \mathrm{~g}$ de gain de poids supplémentaire par gramme de protéines ingérées en faveur du soja, par rapport au sésame. Les protéines de sésame sont donc moins efficaces que celles de soja pour la croissance.

\section{DISCUSSION}

\section{A. - Influence du taux de protéines}

Au cours de nos différentes expériences, nous trouvons une amélioration de la vitesse de croissance des animaux avec le taux de protéines de la ration jusqu'au seuil de I7-I 8 p. Ioo de protéines. A l'inverse, SMITH et al. (I960) n'enregistraient pas d'amélioration au-delà de 13-I4 p. Ioo de protéines. Pour tous les régimes, nous avons enregistré des indices de consommation moyens beaucoup plus faibles que ces auteurs $(2,9$ à 3,6 contre 3,6 à 4,3). Ceci peut correspondre à une meilleure qualité des protéines de soja ou de sésame, par rapport au mélange de protéines complexe et variable utilisé par Smith, DONEFER et MATHIEU (luzerne, trèfle, maïs, orge, avoine et soja). D'autre part, HECKMANN et MEHNER (I970) ont enregistré des indices de consommation semblables aux nôtres mais avec un mélange complexe de protéines comprenant une fraction non négligeable de farine de poisson. Ces derniers auteurs enregistrent une amélioration significative des performances des lapins entre I3-I4 p. Ioo et I8-20 p. Ioo de protéines. En revanche, ils observent une amélioration significative de l'indice de consommation entre I8-20 p. Ioo de protéines et 27-28 p. IOO, alors que dans nos essais, l'indice de consommation n'est pas modifié au-delà de $20 \mathrm{p}$. Ioo de protéines de soja. Toutefois ces considérations doivent être tempérées par le fait que les différents régimes employés par les auteurs précités et par nous-mêmes n'étaient ni iso-énergétiques ni iso-cellulosiques. Ces travaux doivent donc être considérés comme une simple approche du problème. L'utilisation de rations à taux variable d'une même protéine (soja ou sésame) nous permet cependant de préciser le niveau optimum de ces protéines utilisées seules. Ce taux est du même ordre de grandeur pour les 2 protéines employées ; il est possible qu'il corresponde à la zone dans laquelle il faut rechercher le taux optimum d'un mélange de protéines plus ou moins complexe mais parfaitement équilibré par rapport au besoin.

Sous cet angle, le soja s'avère probablement mieux équilibré que le sésame, puisqu'il permet d'obtenir une efficacité protidique meilleure que ce dernier, à tous les taux azotés envisagés. Cependant, cette remarque doit être nuancée car, pour un même taux azoté, le régime soja est plus riche en énergie (plus d'amidon, moins 
de cendres). A propos du coefficient d'efficacité protidique nous devons souligner enfin que nous retrouvons chez le lapin la diminution classique de l'efficacité protéique associée à une augmentation du taux protéique de la ration à partir d'un optimum (RÉRAT, I97I).

\section{B. - Apports d'acides aminés}

L'étude de la composition en acides aminés de nos rations (tabl. 4) permet de constater qu'avec des aliments donnant une bonne croissance (plus de I7 p. roo de protéines), nous avons apporté plus d'arginine - acide aminé indispensable chez le Lapin (ADAMSON et FISHER, I97I) - que ce qui était recommandé par CHEEKE (I97I) ainsi que par MC WARd, Nicholson et Poulton (I967). En effet, l'optimum fixé par ces auteurs se situe aux environs de o,90 p. Ioo d'arginine dans la ration, et nous en apportons au moins I,04 p. Ioo avec le soja et 2,31 p. Ioo avec le sésame.

Pour la méthionine, ou plus exactement pour les acides aminés soufrés, nous obtenons des résultats comparables à ceux de CHEEKE (I97I), avec des performances optimales pour 0,45 p. Ioo de cystine + méthionine.

Par contre, ChEFkE a fixé aux environs de 0,93 p. Ioo de la ration le besoin en lysine. Si cet apport est fourni par les rations satisfaisantes à base de soja, avec les protéines de sésame nous n'apportons que 0,54 p. Ioo de lysine. Le niveau satisfaisant des performances de croissance obtenues $(34-35 \mathrm{~g} / \mathrm{j}$ avec un indice de consommation de 3,4 à 3,6) nous permet de penser que les évaluations de CHEEKE (I97I) sont probablement largement surestimées bien que cet auteur ne donne ces valeurs qu'avec beaucoup de réserves.

En conclusion, dans les conditions expérimentales décrites, le taux optimum de protéines pour le lapin en croissance se situe aux environs de I7-I 8 p. roo de la ration, tant pour le soja que pour le sésame. D'autre part, les besoins en lysine du Lapin, semblent plus faibles que ceux estimés par CHEEKE en I97I.

Rę̧ pour publication en août 1972.

SUMMARY

GROWTH PERFORMANCES OH THE RABBIT AS AFFECTED BY THE PROTEIN LEVEI, OF SOYBEAN OR SESAME DIETS

During the course of 3 successive experiments and using 170 growing rabbits, we estimated the efficiency of diets with different protein levels. The protein source of all diets was composed of either soybcan oil-meal (I I to 29 p. I 00 proteins) or sesame oil-meal (I 3 to 25 p. I oo proteins). For the two types of proteins, growth was improved till a threshold of $17-18$ p. Ioo proteins. In the case of soybean, the food conversion ratio was improved until a level of I8-20 p. I0O proteins. On the contrary, as regards sesame oil-meal, the food conversion ratio was almost constant for protein levels varying between $I_{3}$ and $25 \mathrm{p}$. I 00 . Analysis of the amino acid composition of our experimental diets shows that the lysine requirement data reported in the litterature are probably widely overestimated. 


\section{RÉFÉRENCES BIBLIOGRAPHIQUES}

Adamson I., Fisher H., I97I. "The amino-acid requirements of the growing rabbit : qualitative needs." Nutr. Rpts. Int., 8, 59-64.

Bradfifld R. B., Maynard L. A., 1958. The protein and calorie efficiency of rabbits. Brit. J. Nutr., 12, 13-17.

Chenke P. R., I97I. Arginine, lysine and methionine needs of the growing rabbit. Nutr. Rpts. Int., 3, $123-128$.

Gamax E., Fisher H., I970. The essentiality of arginine, lysine and methionine for the growing rabbit. Nutr. Rpts. Int., 1, 57-64.

Heckmann F. W., Menner A., 1970. Versuch über den Eiweiss- und Rohfasergehalt im Alleinfutter fur Jungmastkaninchen. Arch. Geflïgelz. Kleintierkd., 19, 29-43.

Hofmann li., Steiner K., r959. Die Wirkung einer eiweissintensiven Fütterung auf die Entwicklung der Jungkaninchen bei kleinen, mittleren und grossen Rassen. Arch. Geflügelz. Kleintierkd., 8, 266-286.

Lebas F., I968. Mesure quantitative de la production laitière chez la lapine. Ann. Zootech., 17, 169-182.

Mc Ward G. W., Nicholson L. B., Poulton B. R., 1967. Arginine requirement of the young rabbit. J. Nutr., 92, II8-120.

RÉRAT A., I97I. La valeur biologique des protéines : quelques acquisitions récentes. Ann. Zootech., 20, I93-246.

Smith S. İ., Donefer E., Mathiev L. G., i960. Protein for growing rabbits. Feed age, 10, 7, $52-54$. 